\title{
In vitro pharmacological interaction of caffeine and first-line antibiotics is antagonistic against clinically important bacterial pathogens
}

\author{
Olufunmiso O. Olajuyigbe ${ }^{1,2 \bowtie}$, Morenike O. Adeoye-Isijola1, Victoria Okon', \\ Otunola Adedayo' ${ }^{1}$ and Roger M. Coopoosamy ${ }^{2}$
}

1Biosciences \& Biotechnology Department, Babcock University, PMB 4005, Ilisan Remo, Ogun State, Nigeria; ${ }^{2}$ Department of Nature Conservation, Mangosuthu University of Technology, Durban, KwaZulu-Natal, South Africa

The in vitro antibacterial activity of pure caffeine powder and its interaction with first line antibiotic against bacterial isolates were investigated with the macrobroth dilution and the checkerboard assay methods. This study showed that caffeine and the antibiotics exhibited various degrees of antibacterial activities. While caffeine had MICs ranging between 67.19 and $268.75 \mu \mathrm{g} / \mathrm{ml}$, chloramphenicol was characterized by MICs between 0.98 and $31.25 \mu \mathrm{g} / \mathrm{ml}$, kanamycin - 15.63-62.5 $\mu \mathrm{g} / \mathrm{ml}$, nalidixic acid $-0.49-250 \mu \mathrm{g} / \mathrm{ml}$, erythromycin $-0.49-62.5 \mu \mathrm{g} /$ $\mathrm{ml}$, tetracycline - 1.99-62.5 $\mu \mathrm{g} / \mathrm{ml}$ and metronidazole - 15.63-31.25 $\mathrm{\mu g} / \mathrm{ml}$. Combining $1 / 2$ MICs and MICs of caffeine with the antibiotics as well as direct combination of caffeine and the antibiotics resulted in significant reduction of antibiotics' effectiveness. The fractional inhibitory concentration index $(\mathrm{FICl})$ for the combination of $1 / 2$ MICs of caffeine with different antibiotics showed antagonistic interactions with the antibiotics except kanamycin which had additive and indifferent interactions with caffeine. The FICI of the MICs of caffeine combined with antibiotics showed a reduction in the number of antagonistic interactions as chloramphenicol, nalidixic acid and erythromycin showed some indifferent interactions while kanamycin was the only antibiotic that showed indifferent interaction against all the bacterial isolates. The direct combination of caffeine and the antibiotics resulted in significant antagonistic interactions higher than in the case when caffeine, at the $1 / 2$ MICs and MICs, was combined with the antibiotics. Although caffeine demonstrated significant antibacterial activity against the selected bacterial isolates, its combination with the selected antibiotics resulted in significant antagonistic interactions. Caffeine should not be combined with antibiotics as this could result in serious therapeutic failure and, possibly, drug toxicity in vivo.

Key words: antibacterial activity, bacterial isolates, caffeine, antibiotics, antagonistic effects

Received: 27 May, 2016; revised: 07 August, 2016; accepted: 09 August, 2016; available on-line: 13 April, 2016

e-mail: funmijuyigbe12@yahoo.com

Abbreviations: FIC, fractional inhibitory concentration; MICs, minimum inhibitory concentrations

\section{INTRODUCTION}

Caffeine, 3,7-dihydro-1,3,7-trimethyl-1H-purine-2,6-dione (Yen et al., 2005), is a white powdered, water soluble plant alkaloid found in many plant species. This psychoactive, widely consumed as a stimulant (Belitz et al., 2009;
Mohanpuria et al., 2010), is a purine alkaloid present in almost 100 plant species (Ashihara, 2006). Natural sources of caffeine include cocoa (DeVries et al., 1981), coffee (Minamisawa et al., 2004) and tea (Najafi et al., 2004). The caffeine content in fresh coffee ranged between 314 and $646 \mathrm{mg} / \mathrm{l}$ (Rodrigues et al., 2007) and the content for brewed tea including black tea $(30.97 \mathrm{mg} / \mathrm{g})$, green tea $(18.70 \mathrm{mg} / \mathrm{g})$ and oolong tea $(23.89 \mathrm{mg} / \mathrm{g})$ was reported by Guo and coworkers (2011), whereas caffeine content in cocoa powder was between 0.66 and 0.71 $\mathrm{mg} / \mathrm{g}$ (Li et al., 2012). Caffeine, a white odorless powder with a bitter taste, has a molecular weight of $194.191 \mathrm{~g} /$ mol, a density of $1.2 \mathrm{~g} / \mathrm{cm}^{3}$ and $\mathrm{pH}$ of 6.9 . The solubility of caffeine in water is about $21.7 \mathrm{mg} / \mathrm{ml}$ at $25^{\circ} \mathrm{C}$, $180 \mathrm{mg} / \mathrm{ml}$ at $80^{\circ} \mathrm{C}$ and $670 \mathrm{mg} / \mathrm{ml}$ at $100^{\circ} \mathrm{C}$. Its boiling point is at $178^{\circ} \mathrm{C}$ and its melting point is at $238^{\circ} \mathrm{C}$ (Florey et al., 1989; Agyemang-Yeboah et al., 2013).

Caffeine has long been known to have numerous effects on human health (Cano-Marquina et al., 2013). In humans, it is mainly metabolized into paraxanthine $(84 \%)$, theobromine $(12 \%)$ and theophylline (4\%) (Lelo et al., 1986; Etherton \& Kochar, 1993). The daily average intake of caffeine through coffee consumption in the US is between 165 and $200 \mathrm{mg} /$ day (Mitchell et al., 2014), with adults taking about $2.4 \mathrm{mg}$ per $\mathrm{kg}$ per day and children aged between 5 and 18 years old taking 1.1 mg per kg per day (Chou et al., 1992; Desbrow et al., 2007), while its daily intake in about four to nine cups of coffee is equivalent to 500 to $600 \mathrm{mg}$. This is recognized as an abuse posing serious health risks known as caffeinism which could result in caffeine dependency, headaches, restlessness, heart palpitations, nervousness, insomnia, vomiting, nausea and diarrhea (James et al., 1985), stimulation of the central nervous system (Nehlig et al., 1992) as well as the release of the stress hormones. When these hormones are chronically elevated, mental concentration is short-lived and fine motor coordination is impaired (Jacobson \& Thurman-Lacey, 1992), the immune system is suppressed, digestion and elimination are impaired and body's normal repair mechanisms are inhibited, thereby accelerating aging process (Hill, 1991; Raber, 1998; Leonard, 2000). When stimulatory effects of caffeine are reduced significantly, there is tolerance adaptation. Due to adaptive reactions to caffeine, individuals become more sensitized to adenosine. A reduction in caffeine consumption effectively increases the normal functional effects of adenosine. This could result in undesirable withdrawal indications in tolerant users. Withdrawal symptoms may appear within 12 to $24 \mathrm{~h}$ after termination of caffeine intake. They may last for 
a maximum of five days, which is the sufficient time, required for the adenosine receptors in the brain to revert to normal levels while mental awareness and general body coordination are restored.

Pharmacologically, caffeine inhibits muscle contraction (Youn et al., 1991), acts as the antagonist of the adenosine receptors to increase their plasmatic concentration (Conlay et al., 1997), potentiates the lethal effects of ionizing radiation which is important during the treatment of cancer (Sakurai et al., 1999), alters glucose metabolism (Greer et al., 2001), sensitizes calcium liberation channels and inhibits phosphodiesterase enzymes (Daly, 2007). Pure caffeine has antibacterial effect (Ramanaviciene et al., 2003a; Cogo et al., 2008; Mohammed \& Al-Bayati, 2009), decreases the adherence of Streptococcus mutants to dental surface (Namboodiripad et al., 2007) and acts as the first dietary component able to protect one against Alzheimer's disease, Parkinson's disease and Huntington's disease (Ross et al., 2000; Lindsay et al., 2002; Ribeiro \& Sebastiao, 2010). It increases the concentration of some immunocompetent cells and reinforces the activity of lysozyme to boost immunity against bacterial pathogens (Ramanaviciene et al., 2002; Ramanaviciene et al., 2003b; Vinod \& Rangari, 2004). Although there are interactions between $\mathrm{A} 2 \mathrm{~A}$ receptors and the dopaminergic system in the brain, as adenosine hinders dopaminergic neurotransmission, the inhibition of $\mathrm{A} 2 \mathrm{~A}$ receptors by caffeine may accelerate dopaminergic activity and worsen psychotic symptoms (Kruger et al., 1996) and as one of the 10 most frequently administered drugs in neonatal intensive care, caffeine is used to correct irregular heartbeat and treat apnea in premature newborns (Funk et al., 2009).

There are 82 known drug interactions with caffeine (Prescription drugs/caffeine interactions, 2016. http:// www.caffeineinformer.com/caffeine-drug-interactions. Accessed 01-05-2016). Caffeine increases the activity of some pain killers such as acetaminophen, tylenol and aspirin. It enhances their effectiveness and brings quicker relief by allowing rapid absorption of these drugs into the body. As a result, many over-the-counter headache drugs contain caffeine in their formula. Its combination with ergotamine in the treatment of migraine and headache clusters helps overcome the drowsiness caused by antihistamines (Nehlig et al., 2000). While caffeine works synergistically with some pain relievers (Hidron et al., 2007), it hinders the proper functioning of other drugs like fluvoxamine, which impedes the action of liver enzyme responsible for the breakdown of caffeine and, thus, increases the central effects and blood concentration of caffeine 5 times (Hidron et al., 2007). Combining caffeine and ephedrine, which are both stimulant drugs, could result in side effects and heart problems. Taking antibiotics along with caffeine can increase the risk of having side effects (Coso et al., 2012), fluoroquinolones decrease the rate of caffeine breakdown by the organism (Hidron et al., 2007). As there is the dearth of information on the in vitro antibacterial action of pure caffeine powder and its interaction with first line antibiotics, this study investigated the effects of pure caffeine in the form of powder on the antibacterial activity of some first line antibiotics against bacterial isolates.

\section{MATERIALS AND METHODS}

Test drugs. The pure powder of caffeine was obtained from the Mangosuthu University of Technology, Durban, South Africa while the bacterial isolates and the pure powder of metronidazole, tetracycline, kanamycin, nalidixic acid, chloramphenicol and erythromycin were obtained from the University of Fort Hare, Alice, South Africa.

Test organisms. The organisms used in the study were obtained from the University of Fort Hare, Alice, South Africa and included Pseudomonas aeruginosa ATCC 15442, Proteus vulgaris CSIR 0030, Escherichia coli ATCC 8739, Bacillus cereus ATCC 10702, Shigella sonnei ATCC 29930, Plesiomonas shigellosis ATCC 379003, Klebsiella pneumoniae ATCC 10031, Pseudomonas aeruginosa ATCC 19582, Enterococcus faecalis ATCC 29212 and Enterobacter cloacae ATCC 13047.

Preparation of the samples. The antibiotics were prepared according to manufacturers' specification. Stock solutions of each of the drugs: kanamycin, tetracycline, chloramphenicol, nalidixic acid, erythromycin and caffeine, were prepared by dissolving each drug in its respective solvent.

Determination of the minimum inhibitory concentrations (MICs). The minimum inhibitory concentrations (MICs) of the caffeine and those of each of the antibiotics were determined by macrobroth dilution bioassay (Olajuyigbe \& Afolayan, 2012). Overnight cultures were diluted with sterile nutrient broth. To determine the MICs of each of the drugs alone, one milliliter of stock solution of caffeine was serially diluted in sterile double strength Mueller Hinton broth in test tubes to obtain concentrations ranging between 1.05 and $537.5 \mu \mathrm{g} / \mathrm{ml}$ while concentrations ranging between 0.488 and $250 \mu \mathrm{g} /$ $\mathrm{ml}$ were prepared for all the antibiotics. To determine the influence of the minimum inhibitory concentrations of caffeine on the antibacterial activities of the antibiotics, different concentrations of each of the antibiotics were serially prepared in double strength Mueller Hinton broth while the minimum inhibitory concentration (MICs) and half MICs $(1 / 2$ MICs) of caffeine were added to respective tubes containing different concentrations of each antibiotic. For the combination of caffeine and the antibiotics, equal volumes of stock solutions of caffeine and each antibiotic were combined before being serially diluted to concentrations corresponding to the MICs of each drug in double strength Mueller Hinton broth. After the drug preparations were serially diluted, $100 \mu \mathrm{l}$ of each diluted bacterial culture was aseptically dispensed to the test tubes containing caffeine alone, antibiotics alone and their combinations and incubated at $37^{\circ} \mathrm{C}$ for $24 \mathrm{~h}$. Bacterial growth was indicated by the turbidity of the test tube. The MIC values were recorded as the lowest concentration of each antibiotic showing no visible growth.

Checkerboard assay. The interactions between caffeine and antibiotics were determined using the checkerboard assay. The drug concentrations used in the checkerboard assay covered the MIC for each drug used in the assay. The fractional inhibitory concentration (FIC) was derived from the lowest concentration of the caffeine and the antibiotic used in combination and resulting in no visible growth of the test organisms in the MuellerHinton broth after incubation for $24 \mathrm{~h}$ at $37^{\circ} \mathrm{C}$ (Mandal et al., 2004). FIC indices (FICI) were calculated using the formula: FIC index = (MIC of caffeine in combination $/$ MIC of caffeine alone) + (MIC of antibiotic in combination/MIC of antibiotic alone). In antimicrobial combination, Eliopoulos and Eliopoulos and coworkers )1988) and Petersen et al., (2006) defined synergy as $\Sigma F I C \leq 0.5$, additivity as $0.5<\Sigma F I C \leq 1$, indifference as $1<\Sigma F I C \leq 4$ and antagonism as $\Sigma F I C>4$. This implies that synergy 
Table 1. Minimum inhibitory concentrations of caffeine and of different antibiotics used alone

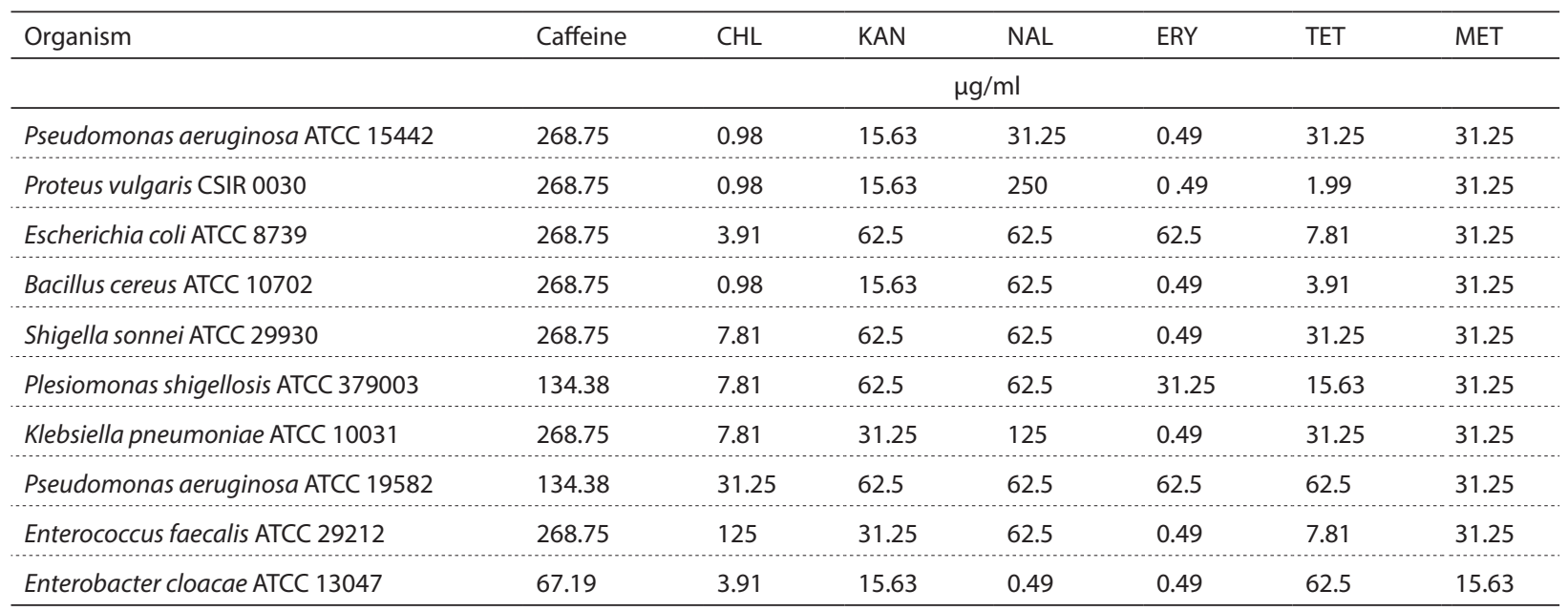

Abreviations: CHL, Chloramphenicol; KAN, Kanamycin; NAL, Nalidixic acid; ERY, Erythromycin; TET, Tetracycline; MET, Metronidazole

determined by the checkerboard method may be defined as $\mathrm{FIC} \leq 0.5$ or $\mathrm{FIC} \leq 1$.

\section{RESULTS}

In this study, we determined the antibacterial activity of caffeine and first line antibiotics as well as the influence of caffeine on the antibacterial activity of the antibiotics. Table 1 shows the minimum inhibitory concentrations for caffeine and antibiotics used alone against the selected bacterial isolates. The caffeine and the antibiotics exhibited various degree of antibacterial activity. Caffeine had MICs ranging between 67.19 and 268.75 $\mu \mathrm{g} / \mathrm{ml}$, chloramphenicol had MICs of between 0.98 and $31.25 \mu \mathrm{g} / \mathrm{ml}$, kanamycin - 15.63-62.5 $\mu \mathrm{g} / \mathrm{ml}$, nalidixic acid - 0.49-250 $\mu \mathrm{g} / \mathrm{ml}$, erythromycin - 0.49-62.5 $\mu \mathrm{g} /$ $\mathrm{ml}$, tetracycline $-1.99-62.5 \mu \mathrm{g} / \mathrm{ml}$ and metronidazole $15.63-31.25 \mu \mathrm{g} / \mathrm{ml}$. At $268.75 \mu \mathrm{g} / \mathrm{ml}$, caffeine inhibited the growth of the bacterial isolates except Pl. shigellosis ATCC 379003 and Ps. aeruginosa ATCC 15442 which were inhibited at $134.38 \mu \mathrm{g} / \mathrm{ml}$ and E. cloacae ATCC 13047 which was inhibited at a concentration of 67.19 $\mu \mathrm{g} / \mathrm{ml}$. At $0.98 \mu \mathrm{g} / \mathrm{ml}$, chloramphenicol inhibited Ps. aeruginosa ATCC 15442, P. vulgaris CSIR 0030 and B. cereus ATCC 10702. At $3.91 \mu \mathrm{g} / \mathrm{ml}$, E. coli ATCC 8739 and E. cloacae ATCC 13047 were inhibited. At $7.81 \mu \mathrm{g} / \mathrm{ml}$, S. sonnei ATCC 29930, Pl. shigellosis ATCC 379003 and K. pneumoniae ATCC 10031 were inhibited. At 31.25 $\mu \mathrm{g} / \mathrm{ml}$, the drug inhibited the growth of Ps. aeruginosa ATCC 15442 and E. faecalis was inhibited at $125 \mu \mathrm{g} / \mathrm{ml}$. Kanamycin, on the other hand, inhibited Ps. aeruginosa ATCC 19582, P. vulgaris CSIR 0030, E. cloacae and B. cereus ATCC 10702 at a concentration of $15.63 \mu \mathrm{g} / \mathrm{ml}$. At $62.5 \mu \mathrm{g} / \mathrm{ml}$, E. coli ATCC 8739, S. sonnei ATCC 29930, Pl. shigellosis ATCC 379003 and Ps. aeruginosa ATCC 15442 were inhibited. At $31.25 \mu \mathrm{g} / \mathrm{ml}$, E. faecalis and $K$. pneumoniae ATCC 10031 were inhibited. Nalidixic acid inhibited the growth of most organisms at $62.5 \mu \mathrm{g} / \mathrm{ml}$

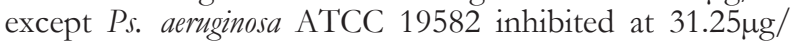
$\mathrm{ml}, P$. vulgaris CSIR 0030 at $250 \mu \mathrm{g} / \mathrm{ml}, K$. pneumoniae ATCC 10031 at $125 \mu \mathrm{g} / \mathrm{ml}$ and E. cloacae ATCC 13047 at $0.49 \mu \mathrm{g} / \mathrm{ml}$. Erythromycin inhibited the growth of most of the organisms at $0.49 \mu \mathrm{g} / \mathrm{ml}$ but Ps. aeruginosa ATCC 15442 and E. coli ATCC 8739 were inhibited at $62.5 \mu \mathrm{g} / \mathrm{ml}$ and Pl. shigellosis ATCC 379003 was inhibited at $31.25 \mu \mathrm{g} / \mathrm{ml}$. Tetracycline inhibited the growth of $P_{s}$. aeruginosa ATCC 19582, S. sonnei ATCC 29930, K. pneumoniae ATCC 10031 at $31.25 \mu \mathrm{g} / \mathrm{ml}$, Ps. aeruginosa ATCC 15442 and E. cloacae ATCC 13047 were inhibited at 62.5 $\mu \mathrm{g} / \mathrm{ml}$, E. faecalis ATCC 29212 and E. coli ATCC 8739 was inhibited at $7.8 \mu \mathrm{g} / \mathrm{ml}$, Pl. shigellosis ATCC 379003 was inhibited at $15.63 \mu \mathrm{g} / \mathrm{ml}, P$. vulgaris CSIR 0030 was inhibited at $1.99 \mu \mathrm{g} / \mathrm{ml}$ and B. cereus ATCC 10702 was inhibited at $3.91 \mu \mathrm{g} / \mathrm{ml}$. Metronidazole inhibited all of the bacterial isolates at $31.25 \mu \mathrm{g} / \mathrm{ml}$ except E. cloacae being inhibited at $15.63 \mu \mathrm{g} / \mathrm{ml}$.

Table 2 shows the influence of caffeine at half of the minimum inhibitory concentrations $(1 / 2$ MICs) on the antibacterial activity of the antibiotics. Combining caffeine with the antibiotics at its half MICs resulted in significant increase of the MICs of the antibiotics. Chloramphenicol that inhibited the bacterial isolates at concentrations ranging between 0.98 and $125 \mu \mathrm{g} / \mathrm{ml}$ when used alone had MICs ranging between 3.91 and $250 \mu \mathrm{g} /$ $\mathrm{ml}$ when used in combination with caffeine at its half MICs. At $31.25 \mu \mathrm{g} / \mathrm{ml}$, this antibiotic inhibited the growth of P. vulgaris CSIR 0030 and E. coli ATCC 8739. However, Ps. aeruginosa ATCC 19582 and B. cereus ATCC 10702 were inhibited at $3.91 \mu \mathrm{g} / \mathrm{ml}$, S. sonnei ATCC 29930, K. pneumonia ATCC 10031, Ps. aeruginosa ATCC 15442 and E. faecalis ATCC 29212 at $250 \mu \mathrm{g} / \mathrm{ml}$ while P. shigellosis ATCC 379003 and E. cloacae were inhibited at $125 \mu \mathrm{g} / \mathrm{ml}$.

Kanamycin having MICs ranging between 15.63 and $62.5 \mu \mathrm{g} / \mathrm{ml}$ when used alone had its MICs ranging between 1.95 and $62.5 \mu \mathrm{g} / \mathrm{ml}$ when combined with caffeine at its $1 / 2$ MICs. It inhibited the growth of $P$. vulgaris CSIR 0030 and E. coli ATCC 8739 at $1.95 \mu \mathrm{g} / \mathrm{ml}$, K. pneumoniae ATCC 10031 and E. faecalis ATCC 29212 at $15.63 \mu \mathrm{g} / \mathrm{ml}$, P. shigellosis ATCC 379003 and E. cloacae ATCC 13047 were inhibited at $31.25 \mu \mathrm{g} / \mathrm{ml}$ while Ps. aeruginosa ATCC 15442 and Ps. aeruginosa ATCC 19582 were inhibited at $62.5 \mu \mathrm{g} / \mathrm{ml}$. While nalidixic acid used alone had MICs ranging between 0.49 and $250 \mu \mathrm{g} /$ $\mathrm{ml}$, its combination with caffeine $(1 / 2$ MICs) resulted in its inhibitory concentrations, effective against all the test bacterial isolates, being greater than $250 \mu \mathrm{g} / \mathrm{ml}$. On the other hand, erythromycin had MICs of 0.49 to $62.5 \mu \mathrm{g} / \mathrm{ml}$ when used alone with most of the isolates being inhibited at $0.49 \mu \mathrm{g} / \mathrm{ml}$ and it had its inhibitory 
Table 2. Caffeine at half of its minimum inhibitory concentrations $\left(1 /{ }_{2} \mathrm{MIC}\right)$ in combination with the different antibiotics

\begin{tabular}{|c|c|c|c|c|c|c|}
\hline Organism & $\mathrm{CHL} / \mathrm{CAF}$ & $\mathrm{KAN} / \mathrm{CAF}$ & $\mathrm{NAL} / \mathrm{CAF}$ & $\mathrm{ERY} / \mathrm{CAF}$ & TET/CAF & MET/CAF \\
\hline & \multicolumn{6}{|c|}{$\mu \mathrm{g} / \mathrm{ml}$} \\
\hline Pseudomonas aeruginosa ATCC 15442 & $3.91 / 134.8$ & $62.5 / 134.8$ & $>250 / 134.8$ & $62.5 / 134.8$ & $15.63 / 134.8$ & $125 / 134.8$ \\
\hline Proteus vulgaris CSIR 0030 & $31.25 / 134.8$ & $1.95 / 134.8$ & $>250134.8$ & $62.5 / 134.8$ & $7.81 / 134.8$ & $125 / 134.8$ \\
\hline Escherichia coli ATCC 8739 & $31.25 / 134.8$ & $1.95 / 134.8$ & $>250 / 134.8$ & $125 / 134.8$ & $15.63 / 134.8$ & $250 / 134.8$ \\
\hline Bacillus cereus ATCC 10702 & $3.91 / 134.8$ & $7.81 / 134.8$ & $>250 / 134.8$ & $62.5 / 134.8$ & $15.63 / 134.8$ & $62.5 / 134.8$ \\
\hline Shigella sonnei ATCC 29930 & $250 / 134.8$ & $7.81 / 134.8$ & $>250 / 134.8$ & $62.5 / 134.8$ & $125 / 134.8$ & $125 / 134.8$ \\
\hline Plesiomonas shigellosis ATCC 379003 & $125 / 134.8$ & $31.25 / 134.8$ & $>250 / 134.8$ & $125 / 134.8$ & $62.5 / 134.8$ & $125 / 134.8$ \\
\hline Klebsiella pneumoniae ATCC 10031 & $250 / 134.8$ & $15.63 / 134.8$ & $>250 / 134.8$ & $125 / 134.8$ & $125 / 134.8$ & $125 / 134.8$ \\
\hline Pseudomonas aeruginosa ATCC 19582 & $250 / 67.4$ & $62.5 / 67.4$ & $>250 / 67.4$ & $125 / 67.4$ & $62.5 / 67.4$ & $125 / 67.4$ \\
\hline Enterococcus faecalis ATCC 29212 & $250 / 134.8$ & $15.63 / 134.8$ & $>250 / 134.8$ & $250 / 134.8=$ & $125 / 134.8$ & $62.5 / 134.8$ \\
\hline Enterobacter cloacae ATCC 13047 & $125 / 37.4$ & $31.25 / 37.4$ & $>250 / 37.4$ & $125 / 37.4$ & $62.5 / 37.4$ & $62.5 / 37.4$ \\
\hline
\end{tabular}

Abbreviations: The combination of caffeine and antibiotics. CHL, Chloramphenicol; KAN, Kanamycin; NAL, Nalidixic acid; ERY, Erythromycin; TET, Tetracycline; MET, Metronidazole; CAF, Caffeine

Table 3. Minimum inhibitory concentrations (MICs) of caffeine in combination with different antibiotics

\begin{tabular}{llccccc}
\hline Organism & CHL/CAF & KAN/CAF & NAL/CAF & ERY/CAF & TET/CAF & MET/CAF \\
\hline & & & & $\mu \mathrm{g} / \mathrm{ml}$ & & \\
\hline Pseudomonas aeruginosa ATCC 15442 & $31.25 / 268.75$ & $0.98 / 268.75$ & $250 / 268.75$ & $7.81 / 268.75$ & $7.81 / 268.75$ & $250 / 268.75$ \\
\hline Proteus vulgaris CSIR 0030 & $15.63 / 268.75$ & $1.95 / 268.75$ & $<250 / 268.75$ & $125 / 268.75$ & $15.63 / 268.75$ & $250 / 268.75$ \\
\hline Escherichia coli ATCC 8739 & $62.5 / 268.75$ & $62.5 / 268.75$ & $<250 / 268.75$ & $125 / 268.75$ & $31.25 / 268.75$ & $250 / 268.75$ \\
\hline Bacillus cereus ATCC 10702 & $62.5 / 268.75$ & $31.25 / 268.75$ & $250 / 268.75$ & $<250 / 268.75$ & $31.25 / 268.75$ & $<250 / 268.75$ \\
\hline Shigella sonnei ATCC 29930 & $125 / 268.75$ & $31.25 / 268.75$ & $250 / 268.75$ & $<250 / 268.75$ & $250 / 268.75$ & $<250 / 268.75$ \\
\hline Plesiomonas shigellosis ATCC 379003 & $31.25 / 134.38$ & $125 / 134.38$ & $125 / 134.38$ & $250 / 134.38$ & $7.81 / 134.38$ & $250 / 134.38$ \\
\hline Klebsiella pneumoniae ATCC 10031 & $125 / 268.75$ & $62.5 / 268.75$ & $250 / 268.75$ & $125 / 268.75$ & $125 / 268.75$ & $250 / 268.75$ \\
\hline Pseudomonas aeruginosa ATCC 19582 & $62.5 / 134.38$ & $31.25 / 134.38$ & $250 / 134.38$ & $250 / 134.38$ & $62.5 / 134.38$ & $250 / 134.38$ \\
\hline Enterococcus faecalis ATCC 29212 & $125 / 268.75$ & $62.5 / 268.75$ & $250 / 268.75$ & $250 / 268.75$ & $250 / 268.75$ & $250 / 268.75$ \\
Enterobacter cloacae ATCC 13047 & $31.25 / 67.19$ & $7.81 / 67.19$ & $<250 / 67.19$ & $250 / 67.19$ & $125 / 67.19$ & $125 / 67.19$ \\
\hline
\end{tabular}

Abbreviations: CHL, Chloramphenicol; KAN, Kanamycin; MET, Metronidazole; TET, Tetracycline; NAL, Nalidixic acid; ERY, Erythromycin

Table 4. Antibacterial effects of direct combination of caffeine and the different antibiotics against bacterial isolates

\begin{tabular}{lcccccc}
\hline Organism & CHL/CAF & KAN/CAF & NAL/CAF & ERY/CAF & TET/CAF & MET/CAF \\
\hline & & & & $\mu \mathrm{m} / \mathrm{ml}$ & \\
\hline Pseudomonas aeruginosa ATCC 15442 & $125 / 125$ & $250 / 250$ & $250 / 250$ & $7.81 / 7.81$ & $250 / 250$ & $250 / 250$ \\
\hline Proteus vulgaris CSIR 0030 & $62.5 / 62.5$ & $250 / 250$ & $125 / 125$ & $62.5 / 62.5$ & $125 / 125$ & $250 / 250$ \\
\hline Escherichia coli ATCC 8739 & $125 / 125$ & $125 / 125$ & $125 / 125$ & $125 / 125$ & $125 / 125$ & $250 / 250$ \\
\hline Bacillus cereus ATCC 10702 & $125 / 125$ & $125 / 125$ & $250 / 250$ & $250 / 250$ & $250 / 250$ & $250 / 250$ \\
\hline Shigella sonnei ATCC 29930 & $125 / 125$ & $125 / 125$ & $250 / 250$ & $250 / 250$ & $250 / 250$ & $250 / 250$ \\
\hline Plesiomonas shigellosis ATCC 379003 & $125 / 125$ & $125 / 125$ & $250 / 250$ & $250 / 250$ & $125 / 125$ & $250 / 250$ \\
\hline Klebsiella pneumoniae ATCC 10031 & $125 / 125$ & $125 / 125$ & $250 / 250$ & $250 / 250$ & $250 / 250$ & $250 / 250$ \\
\hline Pseudomonas aeruginosa ATCC 19582 & $125 / 125$ & $125 / 125$ & $250 / 250$ & $250 / 250$ & $125 / 125$ & $250 / 250$ \\
\hline Enterococcus faecalis ATCC 29212 & $125 / 125$ & $250 / 250$ & $250 / 250$ & $250 / 250$ & $125 / 125$ & $250 / 250$ \\
\hline Enterobacter cloacae ATCC 13047 & $125 / 125$ & $125 / 125$ & $250 / 250$ & $250 / 250$ & $250 / 250$ & $250 / 250$ \\
\hline
\end{tabular}

Abbreviations: CHL, Chloramphenicol; KAN, Kanamycin; MET, Metronidazole; TET, Tetracycline; NAL, Nalidixic acid; ERY, Erythromycin 
Table 5. Fractional inhibitory concentrations index of the effects of half of the minimum inhibitory concentrations of caffeine ( $\left.1 /{ }_{2} \mathrm{MICs}\right)$ on the antibacterial activity of the different antibiotics

\begin{tabular}{|c|c|c|c|c|c|c|c|c|c|c|c|c|}
\hline Organism & $\begin{array}{l}\mathrm{CHL} / \\
\mathrm{CAF}\end{array}$ & Rem & $\begin{array}{l}\text { KAN/ } \\
\text { CAF }\end{array}$ & Rem & $\begin{array}{l}\mathrm{NAL/} \\
\mathrm{CAF}\end{array}$ & Rem & $\begin{array}{l}\text { ERY/ } \\
\text { CAF }\end{array}$ & Rem & $\begin{array}{l}\text { TET/ } \\
\text { CAF }\end{array}$ & Rem & $\begin{array}{l}\text { MET/ } \\
\text { CAF }\end{array}$ & Rem \\
\hline & $(\mathrm{FICl})$ & & $(\mathrm{FICl})$ & & $(\mathrm{FICl})$ & & $(\mathrm{FICl})$ & & $(\mathrm{FICl})$ & & $(\mathrm{FICl})$ & \\
\hline $\begin{array}{l}\text { Pseudomonas aeruginosa } \\
\text { ATCC } 15442\end{array}$ & 4.5 & Antag & 4.5 & Antag & 8.5 & Antag & 128.5 & Antag & 1 & Add & 4.5 & Antag \\
\hline Proteus vulgaris CSIR 0030 & 32.5 & Antag & 0.63 & Add & 4.5 & Antag & $128.5 \mathrm{~A}$ & Antag & 4.5 & Antag & 4.5 & Antag \\
\hline Escherichia coli ATCC 8739 & 8.5 & Antag & 0.53 & Add & 4.5 & Antag & 2.5 & Indiff & 2.5 & Indiff & 8.5 & Antag \\
\hline Bacillus cereus ATCC 10702 & 4.5 & Antag & 1.0 & Add & 4.5 & Antag & 128.5 & Antag & 4.5 & Antag & 2.5 & Indiff \\
\hline Shigella sonnei ATCC 29930 & 32.5 & Antag & 0.75 & Add & 4.5 & Antag & 128.5 & Antag & 4.5 & Antag & 4.5 & Antag \\
\hline $\begin{array}{l}\text { Plesiomonas shigellosis } \\
\text { ATCC } 379003\end{array}$ & 16.5 & Antag & 1.0 & Add & 4.5 & Antag & 4.5 & Antag & 4.5 & Antag & 4.5 & Antag \\
\hline $\begin{array}{l}\text { Klebsiella pneumoniae } \\
\text { ATCC } 10031\end{array}$ & 32.5 & Antag & 1.0 & Add & 1.5 & Indiff & 255.5 & Antag & 4.5 & Antag & 4.5 & Antag \\
\hline $\begin{array}{l}\text { Pseudomonas aeruginosa } \\
\text { ATCC } 19582\end{array}$ & 8.5 & Antag & 1.5 & Indiff & 4.5 & Antag & 2.5 & Indiff & 1.5 & Indiff & 4.5 & Antag \\
\hline $\begin{array}{l}\text { Enterococcus faecalis ATCC } \\
29212\end{array}$ & 2.5 & Indiff & 1.0 & Add & 4.5 & Antag & 500.5 & Antag & 8.5 & Antag & 2.5 & Indiff \\
\hline $\begin{array}{l}\text { Enterobacter cloacae ATCC } \\
13047\end{array}$ & 32.5 & Antag & 2.5 & Indiff & 10.5 & Antag & 255.5 & Antag & 1.5 & Indiff & 4.5 & Antag \\
\hline
\end{tabular}

Abbreviations: Add, Additive; Antag, Antagonistic; Indiff, Indifferent; FICl, Fractional inhibitory concentration index; Chl, Chloramphenicol; Kan, Kanamycin; Nal, Nalidixic acid; Ery, Erythromycin; Tet, Tetracycline; Met, Metronidazole; CAF, Caffeine; Rem, Remarks

concentrations increased to $62.5-250 \mu \mathrm{g} / \mathrm{ml}$. Used in the combination, erythromycin inhibited Ps. aeruginosa ATCC 19582, P. vulgaris CSIR 0030, B. cereus ATCC 10702 and S. sonnei ATCC 29930 at $62.5 \mu \mathrm{g} / \mathrm{ml}$, E. coli ATCC 8739, P. shigellosis ATCC 379003, K. pneumoniae ATCC 10031, Ps. aeruginosa ATCC 15442 and E. cloacae at $125 \mu \mathrm{g} / \mathrm{ml}$ and E. faecalis ATCC 29212 at $250 \mu \mathrm{g} / \mathrm{ml}$. Half MICs of caffeine increased the MICs of tetracycline from between 1.99 and $62.5 \mu \mathrm{g} / \mathrm{ml}$ when used alone to $7.81-125$ $\mu \mathrm{g} / \mathrm{ml}$ when in combination. In the combination with caffeine, tetracycline inhibited $P$. vulgaris CSIR 0030 at $7.81 \mu \mathrm{g} / \mathrm{ml}$, Ps. aeruginosa ATCC 19582, E. coli ATCC 8739 and B. cereus ATCC 10702 at $15.63 \mu \mathrm{g} / \mathrm{ml}, P$. shigellosis ATCC 379003, Ps. aeruginosa ATCC 15442 and E. cloacae at $62.5 \mu \mathrm{g} / \mathrm{ml}$ while $K$. pneumoniae ATCC 10031 and E. faecalis ATCC 29212 were inhibited at $125 \mu \mathrm{g} / \mathrm{ml}$. Although the MICs of metronidazole used alone ranged from 15.63 to $31.25 \mu \mathrm{g} / \mathrm{ml}$, they increased to between

Table 6. Fractional inhibitory concentration index of the effect of minimum inhibitory concentrations (MICs) of caffeine on the antibacterial activities of different antibiotics

\begin{tabular}{|c|c|c|c|c|c|c|c|c|c|c|c|c|}
\hline Organism & $\begin{array}{l}\mathrm{CHL} / \\
\mathrm{CAF}\end{array}$ & Rem & $\begin{array}{l}\text { KAN/ } \\
\text { CAF }\end{array}$ & Rem & $\begin{array}{l}\text { NAL/ } \\
\text { CAF }\end{array}$ & Rem & $\begin{array}{l}\mathrm{ERY} / \\
\mathrm{CAF}\end{array}$ & Rem & $\begin{array}{l}\text { TET/ } \\
\text { CAF }\end{array}$ & Rem & $\begin{array}{l}\text { MET/ } \\
\text { CAF }\end{array}$ & Rem \\
\hline & $(\mathrm{FICl})$ & & $(\mathrm{FICl})$ & & $(\mathrm{FICl})$ & & $(\mathrm{FICl})$ & & $(\mathrm{FICl})$ & & $(\mathrm{FICl})$ & \\
\hline $\begin{array}{l}\text { Pseudomonas aeruginosa ATCC } \\
15442\end{array}$ & 33 & Antag & 1.06 & Indiff & 9 & Antag & 17 & Antag & 1.25 & Indiff & 9 & Antag \\
\hline Proteus vulgaris CSIR 0030 & 17 & Antag & 1.13 & Indiff & 2 & Indiff & 256 & Antag & 9 & Antag & 9 & Antag \\
\hline Escherichia coli ATCC 8739 & 17 & Antag & 2 & Indiff & 5 & Antag & 3 & Indiff & 5 & Antag & 9 & Antag \\
\hline Bacillus cereus ATCC 10702 & 65 & Antag & 3 & Indiff & 5 & Antag & 511 & Antag & 9 & Antag & 9 & Antag \\
\hline Shigella sonnei ATCC 29930 & 17 & Antag & 1.5 & Indiff & 5 & Antag & 511 & Antag & 9 & Antag & 9 & Antag \\
\hline $\begin{array}{l}\text { Plesiomonas shigellosis ATCC } \\
379003\end{array}$ & 5 & Antag & 3 & Indiff & 3 & Indiff & 9 & Antag & 1.5 & Indiff & 9 & Antag \\
\hline $\begin{array}{l}\text { Klebsiella pneumoniae ATCC } \\
10031\end{array}$ & 17 & Antag & 3 & Indiff & 3 & Indiff & 256 & Antag & 5 & Antag & 9 & Antag \\
\hline $\begin{array}{l}\text { Pseudomonas aeruginosa ATCC } \\
19582\end{array}$ & 3 & Indiff & 1.5 & Indiff & 5 & Antag & 3 & Indiff & 2 & Indiff & 9 & Antag \\
\hline $\begin{array}{l}\text { Enterococcus faecalis ATCC } \\
29212\end{array}$ & 2 & Indiff & 26 & Antag & 5 & Antag & 511 & Antag & 32 & Antag & 9 & Antag \\
\hline $\begin{array}{l}\text { Enterobacter cloacae ATCC } \\
13047\end{array}$ & 9 & Antag & 1.5 & Indiff & 511 & Antag & 511 & Antag & 3 & Indiff & 9 & Antag \\
\hline
\end{tabular}

Abbreviations: Add, Additive; Antag, Antagonistic; Indiff, Indifferent; FICl, Fractional inhibitory concentration index; Chl, Chloramphenicol; Kan, Kanamycin; Nal, Nalidixic acid; Ery, Erythromycin; Tet, Tetracycline; Met, Metronidazole, CAF, Caffeine, Rem, Remarks 
Table 7. Fractional inhibitory concentration index of the effect of direct combination of caffeine and the different antibiotics against the bacterial isolates

\begin{tabular}{|c|c|c|c|c|c|c|c|c|c|c|c|c|}
\hline Organism & $\begin{array}{l}\mathrm{CHL} / \\
\mathrm{CAF}\end{array}$ & Rem & $\begin{array}{l}\text { KAN/ } \\
\text { CAF }\end{array}$ & Rem & $\begin{array}{l}\mathrm{NAL} / \\
\mathrm{CAF}\end{array}$ & Rem & $\begin{array}{l}\mathrm{ERY} / \\
\mathrm{CAF}\end{array}$ & Rem & $\begin{array}{l}\text { TET/ } \\
\text { CAF }\end{array}$ & Rem & $\begin{array}{l}\text { MET/ } \\
\text { CAF }\end{array}$ & Rem \\
\hline & $\mathrm{FICl}$ & & $\mathrm{FICl}$ & & $\mathrm{FICl}$ & & $\mathrm{FICl}$ & & $\mathrm{FICl}$ & & $\mathrm{FICl}$ & \\
\hline $\begin{array}{l}\text { Pseudomonas aeruginosa ATCC } \\
15442\end{array}$ & 129 & Antag & 257 & Antag & 256 & Antag & 8 & Antag & 256 & Antag & 256 & Antag \\
\hline Proteus vulgaris CSIR 0030 & 64 & Antag & 257 & Antag & 129 & Antag & 64 & Antag & 129 & Antag & 256 & Antag \\
\hline Bacillus cereus ATCC 10702 & 128 & Antag & 128 & Antag & 256 & Antag & 256 & Antag & 256 & Antag & 256 & Antag \\
\hline Shigella sonnei ATCC 29930 & 17 & Antag & 17 & Antag & 33 & Antag & 33 & Antag & 33 & Antag & 33 & Antag \\
\hline $\begin{array}{l}\text { Plesiomonas shigellosis ATCC } \\
379003\end{array}$ & 17 & Antag & 17 & Antag & 34 & Antag & 34 & Antag & 33 & Antag & 34 & Antag \\
\hline $\begin{array}{l}\text { Klebsiella pneumoniae ATCC } \\
10031\end{array}$ & 17 & Antag & 5 & Antag & 33 & antag & 33 & Antag & 33 & Antag & 33 & Antag \\
\hline $\begin{array}{l}\text { Pseudomonas aeruginosa ATCC } \\
19582\end{array}$ & 5 & Antag & 17 & Antag & 10 & Antag & 10 & Antag & 5 & Antag & 10 & Antag \\
\hline $\begin{array}{l}\text { Enterococcus faecalis ATCC } \\
29212\end{array}$ & 2 & Indiff & 3 & Indiff & 2.93 & Antag & 2.93 & Antag & 1.5 & Indiff & 2.93 & Antag \\
\hline $\begin{array}{l}\text { Enterobacter cloacae ATCC } \\
13047\end{array}$ & 34 & Antag & 32 & Antag & 68 & Antag & 68 & Antag & 68 & Antag & 68 & Antag \\
\hline
\end{tabular}

Abbreviations: Add, Additive; Antag, Antagonistic; Indiff, Indifferent; FICl, Fractional inhibitory concentration index; Chl, Chloramphenicol; Kan, Kanamycin; Nal, Nalidixic acid; Ery, Erythromycin; Tet, Tetracycline; Met, Metronidazole; CAF, Caffeine; Rem, Remarks

62.5 and $250 \mu \mathrm{g} / \mathrm{ml}$ when combined with caffeine at its half MICs. Upon the combination of metronidazole with caffeine at $1 / 2$ MICs, E. faecalis ATCC 29212, E. cloacae ATCC 13047 and B. cereus ATCC 10702 were inhibited at $62.5 \mu \mathrm{g} / \mathrm{ml}$ and E. coli ATCC 8739 was inhibited at $250 \mu \mathrm{g} / \mathrm{ml}$ while other bacterial isolates were inhibited at $125 \mu \mathrm{g} / \mathrm{ml}$.

Table 3 shows the influence of the MIC of the caffeine on the antibacterial activity of the antibiotics. In combinations with caffeine, chloramphenicol had MICs ranging between 15.63 and $125 \mu \mathrm{g} / \mathrm{ml}$. It inhibited P. vulgaris CSIR 0030 at $15.63 \mu \mathrm{g} / \mathrm{ml}$, E. cloacae, Ps. aeruginosa ATCC 19582 and P. shigellosis ATCC 379003 at $31.25 \mu \mathrm{g} / \mathrm{ml}$, E. coli, B. cereus ATCC 10702 and Ps. aeruginosa ATCC 15442 at $62.5 \mu \mathrm{g} / \mathrm{ml}$, and S. sonnei ATCC 29930, E. faecalis ATCC 29212, K. pneumoniae ATCC 10031 at $125 \mu \mathrm{g} / \mathrm{ml}$. While the interaction between caffeine and kanamycin resulted in MICs ranging between 0.98 and $62.5 \mu \mathrm{g} / \mathrm{ml}$ for kanamycin, it inhibited Ps. aeruginosa ATCC 19582 at $0.98 \mu \mathrm{g} / \mathrm{ml}$, Proteus vulgaris CSIR 0030 at $1.95 \mu \mathrm{g} / \mathrm{ml}$, E. cloacae at $7.81 \mu \mathrm{g} /$ $\mathrm{ml}$, E. coli ATCC 8739, Ps. aeruginosa ATCC 1542, S. sonnei ATCC 29930 and B. cereus ATCC 10702 at $31.25 \mu \mathrm{g} /$ $\mathrm{ml}$, whereas E. faecalis ATCC 29212 and K. pneumoniae ATCC 10031 at $62.5 \mu \mathrm{g} / \mathrm{ml}$. For nalidixic acid, its MICs ranged between 125 and $>250 \mu \mathrm{g} / \mathrm{ml}$ when combined with caffeine at its MICs. With the exception of P. shigellosis ATCC 379003 being inhibited at $125 \mu \mathrm{g} / \mathrm{ml}$ and E. cloacae ATCC 13047, E. coli ATCC 8739 and P. vulgaris CSIR 0030 being inhibited at concentrations greater than $250 \mu \mathrm{g} / \mathrm{ml}$, other isolates were inhibited at $250 \mu \mathrm{g} /$ $\mathrm{ml}$ when nalidixic acid was combined with MICs of caffeine. Combining erythromycin with caffeine increased the MICs up to $7.81->250 \mu \mathrm{g} / \mathrm{ml}$ with most of the isolates being inhibited at 250 and $>250 \mu \mathrm{g} / \mathrm{ml}$. While the combination of caffeine at MICs with tetracycline resulted in tetracycline's MICs being increased to between 7.81 and $250 \mu \mathrm{g} / \mathrm{ml}$, those of metronidazole was between 125 and $>250 \mu \mathrm{g} / \mathrm{ml}$ with most of the isolates being inhibited at 250 and $>250 \mu \mathrm{g} / \mathrm{ml}$ except E. cloacae ATCC 13047 being inhibited at $125 \mu \mathrm{g} / \mathrm{ml}$. The direct combination of caffeine with the antibiotics resulted in significant increase in the minimum inhibitory concentrations of the antibiotics against all the bacterial isolates as shown in Table 4.

In Tables 5 to 7 , the fractional inhibitory concentration index was used to find out if the interactions between caffeine and the antibiotics were antagonistic, synergistic, additive or indifferent. The fractional inhibitory concentration of the combination of $1 / 2$ MICs of caffeine with the different antibiotics showed that all the antibiotics exhibited antagonistic except kanamycin having additive and indifferent interactions with caffeine as shown in Table 5. However, combining the antibiotics with the MICs of caffeine showed a reduction in number of antagonistic interactions as chloramphenicol, nalidixic acid and erythromycin showed some indifferent while kanamycin was the only antibiotic that showed indifferent interaction against all the selected bacterial strains as shown in Table 6. The direct combination of caffeine and each of the antibiotics resulted in significant antagonistic interactions with inhibitory concentrations higher than when caffeine was combined with the antibiotics at the $1 / 2$ MICs and MICs as shown in Table 7.

\section{DISCUSSION}

It is a common practice to wash down drugs and orally administer medications with caffeine containing soft drinks such as cola and energy drinks. Occasionally, caffeine containing food such as cocoa, coffee and tea is used in beverages to reduce the after taste some bitter drugs would leave after an oral administration. Some of these caffeine containing foods are used to persuade litthe children to take bitter drugs. In pharmaceutical drugs such as ergotamine, caffeine is included for the treatment of migraine headaches and is combined with nonsteroidal anti-inflammatory drugs for relieving pain (Saw- 
ynok, 1995). These practices have continued for ages without exceptional consideration for possible drug-drug or drug-food interactions between the caffeine contained by the food and the different medications, especially antibiotics used in treating bacterial infections. Therefore, it becomes necessary to investigate interactions between caffeine and antibiotics (Kang et al., 2012).

Although several reports showed that caffeine possesses antibacterial properties, most of these studies focused on caffeine containing food. For instance, Ramanaviciene and coworkers (2002) reported antibacterial activity of caffeine against Ps. flourescens and E. coli. Mohammed and Al-Bayati (2009) reported antibacterial activity of caffeine from Coffee arabica (coffee beans) and Camelia sinensis (green tea leaves) against $S$. aureus, B. cereus, E. coli, P. mirabilis and K. pneumoniae. Inhibitory effects of Coffea canephora extracts against L. pneumophila (Furuhata et al., 2002), S. mercescens and E. cloacae (Almeida et al., 2006) and S. mutans (Antonio et al., 2010; Almeida et al., 2012) were also reported while Suárez-Quiroz and coworkers (2004), Wilmot (2006) and Chen and coworkers (2013) showed that caffeine inhibits mold and fungi. However, there is the dearth of information on the antibacterial activities of pure caffeine powder. In this study, the minimum inhibitory concentration of pure caffeine powder ranged between 67.19 and 268.75 $\mu \mathrm{g} / \mathrm{ml}$. While most of the bacterial isolates were inhibited at $268.75 \mu \mathrm{g} / \mathrm{ml}, P$. shigellosis ATCC 379003 and Ps. aeruginosa ATCC 15442 were inhibited at $134.38 \mu \mathrm{g} / \mathrm{ml}$ and E. cloacae ATCC 13047 was inhibited at $67.19 \mu \mathrm{g} /$ $\mathrm{ml}$. These results are in agreement with previous reports. Mohammed and Al-Bayati (2009) reported that MICs of caffeine coffee ranged between 62.5 and $250 \mu \mathrm{g} / \mathrm{ml}$ and green tea caffeine ranged from 62.5 to $500 \mu \mathrm{g} / \mathrm{ml}$ while Pruthviraj and coworkers (2011) reported MICs of caffeine isolated from tea between 65.5 and $250 \mu \mathrm{g} / \mathrm{ml}$ and green tea caffeine between 65.5 and $500 \mu \mathrm{g} / \mathrm{ml}$. The activity of pure caffeine, used in this study, was further corroborated by Ramanaviciene and coworkers (2003b) who indicated that pure caffeine has a direct antibacterial effect.

Furthermore, the interactions of caffeine with antibiotics were previously reported. While Charles and Rawal (1979) indicated that caffeine decreased the antibacterial activity of chloramphenicol and tetracycline hydrochloride, Hosseinzadeh and coworkers (2006) reported that caffeine works synergistically with carbenicillin, ceftizoxime and gentamicin which are effective against $P$ s. aeruginosa and S. aureus. Esinome and coworkers (2008) reported that caffeine combined with ampicillin resulted in indifferent interaction while its combination with benzypenicillin was antagonistic. Kang and coworkers (2012) reported that there are no synergistic interactions between caffeine and bleomycin or cisplatin but the activities of ciprofloxacin were decreased when paired with caffeine. In this study, the combination of caffeine with chloramphenicol, nalidixic acid, erythromycin, tetracycline and metronidazole resulted, mostly, in antagonistic interactions with the exception of the interaction with kanamycin that resulted mostly in either additive or indifferent effects. Kang and coworkers (2012), however, indicated that kanamycin activity is increased upon the addition of $0.25 \mathrm{mg} / \mathrm{ml}(0.025 \% \mathrm{w} / \mathrm{v})$ of caffeine in a previous study.

Caffeine increases the susceptibility of bacteria and higher cells to different antibiotics and DNA-damaging agents when used in pre-treatment (Grigg et al., 1985; Petru et al. 1990; Selby and Sancar, 1990) by intercalating into the DNA (Tornaletti et al., 1989). Although caffeine interacts with bacterial nucleic acids (Sacks \& Thompson, 1977; Kawamukai et al., 1986), inhibits DNA synthesis (Sandlie et al., 1983; Osman \& McCready, 1998) and cause frameshift mutations (Pons \& Muller, 1990), it can also interact with the enzymes responsible for repair of bacterial DNA damage by inhibiting ATP-dependent enzymes (Selby \& Sancar, 1990) and inactivate ataxia-telangiectasia-mutated (ATM) and ATM-and-Rad3-related proteins responsible for the genome stability (Cortez, 2003). While the variety of the effects of combining caffeine and these antibiotics may resulted from the influence of the physicochemical interactions between caffeine and the drugs in vitro, the interactions could have resulted from the formation of complexes between the caffeine and the antibiotics (Veselkov et al., 2002). The complex formation with caffeine through hydrophobic van der Waals interactions (Lachman \& Ravin, 1959; Matha \& adbels, 1982; Kapuscinski \& Kimmel 1993; Larsen et al., 1996) may reduce the in vitro antibacterial activity of the less polar antibiotics. The antagonistic interaction or reduction in the antibacterial activities of most of these antibiotics could also be a result of caffeine and antibiotic molecules competing for the same binding sites on DNA or "intercepting" or "protecting" properties of caffeine (Traganos et al., 1991; Davies et al., 2001) or its ability to stimulate hydrophobic molecules into their dimeric structures (Banerjee et al., 2012). Aminoglycoside antibiotics, such as kanamycin, target the ribosomes and interfere with the fidelity of protein synthesis (Poehlsgaard \& Douthwaite, 2005). The interaction between caffeine and kanamycin could result from the complementary activity of both agents. Caffeine could increase susceptibility to kanamycin and intercalate into DNA (Tornaletti et al., 1989) while kanamycin induces mistranslation of mRNA to protein (Misumi \& Tanaka, 1980) and damages DNA base pairs (Kang et al., 2012). On the other hand, caffeine could increase susceptibility to erythromycin but the putative complexes formed between caffeine and erythromycin possibly prevented both antibacterial agents from reaching their target sites of action.

\section{CONCLUSION}

In conclusion, caffeine demonstrated significant antibacterial activity against the selected bacterial isolates. However, its combination with the selected antibiotics resulted in significant antagonistic interactions against the bacterial isolates indicating that, against usual practice of using caffeine containing food or drinks in oral drug administration, caffeine should not be combined with antibiotics as this could result in serious therapeutic failure and, possibly, drug toxicity in vivo.

\section{REFERENCES}

Agyemang-Yeboah F, Oppong SY (2013) Caffeine: The wonder compound, chemistry and properties. Ghana: Kwame Nkrumah University, MSc thesis

Almeida AAP, Farah A, Silva DAM, Nunan EA, Gloria MBA (2006) Antibacterial activity of coffee extracts and selected coffee chemical compounds against Enterobacteria. J Agric Food Chem 54: 87388743. doi: $10.1021 /$ jf0617317

Almeida AAP, Naghetini CC, Santos VR, Antonio AG, Farah A, Glória MBA (2012) Influence of natural coffee compounds, coffee extracts and increased levels of caffeine on the inhibition of Streptococcus mutans. Food Res Int 49: 459-461. doi: 10.1016/j.foodres.2012.07.026

Antonio AG, Moraes RS, Perrone D, Maria LC, Santos KRN, Iorio NLP, Farah A (2010) Species, roasting degree and decaffeination influence the antibacterial activity of coffee against Streptococcus mutans. Food Chem 118: 782-788. doi: 10.1016/j.foodchem.2009.05.063 
Ashihara H (2006) Metabolism of alkaloids in coffee plants. Brazil J Plant Physiol 18: 1-8. doi: 10.1590/S1677- 04202006000100001

Banerjee S, Verma PK, Mitra RK, Basu G, Pal SK (2012) Probing the interior of self-assembled caffeine dimer at various temperatures. $J$ Fluoresc 22: 753-769. doi: 10.1007/s10895-011-1011-3

Belitz HD, Grosch W, Schieberle P (2009) Food Chemistry: Coffee, Tea, Cocoa. Berlin Heidelberg: Springer-Verlag

Cano-Marquina A, Tarín JJ, Cano A (2013) The impact of coffee on health. Maturitas 75: 7-21. doi: 10.1016/j.maturitas.2013.02.002.

Cano-Marquina A, Tarin JJ, Cano A (2013) The impact of coffee on health. Maturitas 75: 7-21. http://dx.doi.org/10.1016/j.maturitas.2013.02.002

Charles BG, Rawal BD (1979) The combined action of methylxanthines with erythromycin and tetracyclines on Staphylococcus aureus. Microbios Lett 10: 143-147.

Chen, J, Zhang S, Yang X (2013) Control of brown rot on nectarines by tea polyphenol combined with tea saponin. Crop Protection 45: 29-35. Doi: 10.1016/j.cropro.2012.11.006

Chou T. (1992) Wake up and smell the coffee: Caffeine, coffee and medical consequences. Western J Med 157: 544-553

Cogo K, Montan MF, Bergamaschi CdeC, Andrade ED, Rosalen PL, Groppo FC (2008) In vitro evaluation of the effect of nicotine, cotinine and caffeine on oral microorganisms. Can J Microbiol 54: 501508. doi: $0.1139 /$ W08-032

Conlay LA, Conant JA, DeBros F, Wurtman R (1997) Caffeine alters plasma adenosine levels. Nature 389: 136. doi: 10.1038/38160

Coso JD, Munoz-Fernandez VE, Munoz G, Fernandez-Elias VE, Ortega JF, Hamouti N, Barbero JC, Munoz-Guerra J (2012) Effects of a caffeine-containing energy drink on simulated soccer performance. PLOS ONE 7: e31380. doi:10.1371/journal.pone.0031380

Cortez D (2003) Caffeine inhibits checkpoint responses without inhibiting the ataxia-telangiectasia-mutated (ATM) and ATM- and Rad3related (ATR) protein kinases. J Biol Chem 278: 37139-37145. doi: 10.1074/jbc.M307088200

Daly JW (2007) Caffeine analogs: biomedical impact. Cell Mol Life Sci 64: 2153-2169

Davies DB, Veselkov DA, Djimant LN, Veselkov AN (2001) Heteroassociation of caffeine and aromatic drugs and their competitive binding with a DNA oligomer. Eur Biophys J 30: 354-366

Desbrow B, Hughes R, Leveritt M, Scheelings P (2007) An examination of consumer exposure to caffeine from retail coffee outlets. Food Chem Toxicol 45: 1588-1592. doi:10.1016/j.fct.2007.02.020

DeVries JW, Johnson KD, Heroff JC (1981) HPLC determination of caffeine and theobromine content of various natural and red dutch cocoas. Hoppe Seyler's Z Physiol Chem 358: 807-817

Eliopoulos GM, Eliopoulos CT (1988) Antibiotic combinations: should they be tested? Clin Microbiol Rev 1: 139-156.

Esinome CO, Okoye FBC, Nworu CS, Agubata CO (2008). In vitro interaction between caffeine and come penicillin antibiotics against Staphylococcu aureus. Trop J Pharm Res 7: 969-974

Etherton GM, Kochar MS (1993) Coffee facts and controversies. Arch of Fam Med 2: 317-322

Florey K (1986) Analytical profiles of drug substances. In Analytical profiles of drug substances Al-Badr AA, Brewer AG, Brenner SG, Deangelis JN, Mollica AJ eds. p 71. London: Academic Press

Funk GD (2009) Losing sleep over the caffeination of prematurity. $J$ Physiol 587: 5299-5300. doi: 10.1113/jphysiol.2009.182303

Furuhata K, Dogazaki C, Hara M, Furuyama M (2002) Inactivation of Legionella pneumophila by phenol compounds contained in coffee. J Antibact Antifung Agents 30: 291-297

Greer F, Hudson R, Ross R, Graham T (2001) Caffeine ingestion decreases glucose disposal during a hyperinsulinemic - euglycemic clamp in sedentary humans. Diabetes 50: 2349-2354. doi: 10.2337/ diabetes.50.10.2349

Grigg GW, Hall RM, Hart NK, Kavulak DR, Lamberton JA, Lane A (1985) Amplification of the antibiotic effects of the bleomycins and tallysomycins: its dependence on the nature of the variable groups. J Antibiotics 38: 99-110

Suárez-Quiroz ML, González-Rios O, Barel M, Guyot B, Schorr-Galindo S, Guiraud JP (2004) Effect of chemical and environmental factors on Aspergillus ochraceus growth and toxigenesis in green coffee. Food Microbiol 21: 629-634

Guo S, Zhu Q, Yang B, Wang J, Ye B (2011) Determination of caffeine content in tea based on poly(safranine $T$ ) electro active film modified electrode. Food Chem 129: 1311-1314. doi: 10.1016/j.foodchem.2011.05.095

Hidron AI, Edwards JR, Patel J, Horan TC, Sievert DM, Pollock DA, Fridkin SK, National Healthcare Safety Network Team \& Participating National Healthcare Safety Network Facilities (2008) NHSN annual update: antimicrobial-resistant pathogens associated with healthcare-associated infections: annual summary of data reported to the National Healthcare Safety Network at the Centers for Disease Control and Prevention, 2006-2007. Infect Contr Hosp Epidemiol 29: 996-1011. doi: 10.1086/591861

Hill P (1991) It is not what you eat, but how you eat it digestion, lifestyle, nutrition. Nutrition 7: 385-395
Hosseinzadeh H, Bazzaz BS, Sadat MM (2006) In vitro evaluation of methylxanthines and some antibiotics: interaction against Staphylococcus aureus and Pseudomonas aeruginosa. Iranian Biomed J 10: 163-167

Jacobson BH, Thurman-Lacey SR (1992) Effect of caffeine on motor performance by caffeine-naive and familiar subjects. Perceptual and Motor Skills 74: 151-157

James JE, Paull I (1985) Caffeine and human reproduction. Rev Env Health 5: 151-167

Kang TM, Yuan J, Nguyen A, Becket E, Yang H, Miller JH (2012) The aminoglycoside antibiotic kanamycin damages DNA bases in Escherichia coli: caffeine potentiates the DNA-damaging effects of kanamycin while suppressing cell killing by ciprofloxacin in escherichia coli and bacillus anthracis. Antimicrob Agents Chemother 56: 3216-3223. doi: 10.1128/AAC.00066-12

Kapuscinski J, Kimmel M (1993) Thermodynamic model of mixed aggregation of intercalators with caffeine in aqueous solution. Biophys Chem 46: 153-163. doi: 10.1016/0301-4622(93)85022-A

Kawamukai M, Murao K, Utsumi R, Himeno M, Komano T (1986) Cell filamentation in an Escherichia coli K-12 fic mutant caused by theophylline or an adenylate cyclase gene (cya)-containing plasmid. FEMS Microb Lett 34: 117-120. doi: 10.1111/j.1574-6968.1986. tb01360.x

Kruger A (1996) Chronic psychiatric patients' use of caffeine: pharmacological effects and mechanisms. Psychol Reports 78: 915-923. doi: 10.2466/pr0.1996.78.3.915

Lachman LJ, Ravin L (1959). Complexation of benzocaine, procaine, and teracine with caffeine $J$ Am Pharm Ass 45: 120-123.

Larsen RW, Jasuja R, Hetzler RK, Muraoka PT, Andrada VG, Jameson M (1996) Spectroscopic and molecular modeling studies of caffeine complexes with DNA intercalators. Biophys J 70: 43-452. doi: 10.1016/S0006-3495(96)79587-5

Lelo A, Birkett DJ, Robson RA, Miners JO (1986) Comparative pharmokinectics of caffeine and its primary demethylated metabolites paraxanthine, theobromine and theophylline in man. Br J Clin Pharmacol 22: 177-182

Li Y, Feng Y, Zhu S, Luo C, Ma J, Zhong F (2012) The effect of alkalization on the bioactive and flavor related components in commercial cocoa powder. J Food Comp Anal 25: 17-23

Leonard B (2000) Stress, depression and the activation of the immune system. World J Biol Psych 1: 17-25.

Lindsay J, Laurin D, Verreault R, Hebert R, Helliwell B, Hill GB, McDowell I (2002) Risk factors for Alzheimer's disease: a prospective analysis from the Canadian Study of Health and Aging. Am J Epidemiol 156: 445-453.

Mandal S, Mandal MD, Pal NK (2004) Evaluation of combination effect of ciprofloxacin and cefazolin against Salmonella enteric serovar typhi isolates by in vitro methods. Calicut Med J 2: e2.

Matha A, Adbels OH (1982) Complex formation between antihistaminic drugs and caffeine. J Am Pharm Ass 9: 208

Minamisawa M, Yoshida S, Takai N (2004) Determination of biologically active substances in roasted coffees using a diode-array HPLC system. Anal Sci 20: 325-328. doi: 10.2116/analsci.20.325

Misumi M, Tanaka N (1980) Mechanism of inhibition of translocation by kanamycin and viomycin: a comparative study with fusidic acid. Biochem Biophys Res Commun, 92: 647-654

Mitchell DC, Knight CA, Hockenberry J, Teplansky R, Hartman TJ (2014) Beverage caffeine intakes in the US. Food Chem Toxicol 63: 136-142. doi: 10.1016/j.fct.2013.10.042

Mohammed MJ, Al-Bayati FA (2009) Isolation, identification and purification of caffeine from Coffea arabica L. and Camellia sinensis L.: A combination antibacterial study. Int J Green Pharm 3: 52-57. doi: 10.4103/0973-8258.49375

Mohanpuria P, Kumar V, Yadav SK (2010) Tea caffeine: metabolism, functions and reduction strategies. J Food Sci Biotech 19: 275-287. doi: 10/1007/s10068-010-0041-y

Najafi NM, Hamid AS, Afshin RK (2003) Determination of caffeine in black tea leaves by fourier transform infrared spectrometry using multiple linear regression. Microchem J 75: 151-158

Namboodiripad PA, Kori S (2008) Can coffee prevent caries? I Conserv Dentistry 12: 17-21. doi: 10.4103/0972-0707.53336

Nehlig A, Daval JL, Debry G (1992) Caffeine and the central nervous system: mechanisms of action, biochemical, metabolic and psychostimulant effects. Brain Res Rev 17: 139-170. doi: 10.1016/01650173(92)90012-B

Nehlig A, Boyet S (2000) Dose-response study of caffeine effect of cerebral functional activity with a specific focus on dependence. Brain Res 858: 71-77. doi: 10.1016/S0006-8993(99)02480-4

Olajuyigbe OO, Afolayan AJ (2012) In vitro antibacterial and time kill assessment of crude methanolic stem bark extract of Acacia mearnsi De Wild against bacteria in shigellosis. Mol 17: 2103-2118. doi: $10.3390 /$ molecules 17022103

Osman F, McCready S (1998) Differential effects of caffeine on DNA damage and replication cell cycle checkpoints in the fission yeast Schizosaccharomyces pombe. Mol Gen Genet 260: 319-334

Poehlsgaard J, Douthwaite S (2005) The bacterial ribosome as a target for antibiotics. Nat Rev Microbiol 3: 870-881 
Petersen PJ, Labthavikul P, Jones CH, Bradford PA (2006) In vitro antibacterial activities of tigecycline in combination with other antimicrobial agents determined by chequerboard and time-kill kinetic analysis. J Antimicrob Chemother 57: 573-576. doi: 10.1093/jac/dki477

Pons FW, Muller P (1990) Induction of frameshift mutations by caffeine in Escherichia coli K12. Mutag 5: 173-178. doi: 10.1093/mutage/ $/ 5.2 .173$

Prescription drugs/caffeine interactions (2016) http://www.caffeineinformer.com/caffeine-drug-interactions. Accessed 01-05-2016

Pruthviraj P, Suchita B, Shital K, Shilpa K (2011) Evaluation of antibacterial activity of caffeine. Int J Res in Ayuveda Pharm 2: 1354-1357

Raber J (1998) Detrimental effects of chronic hypothalamic-pituitaryadrenal axis activation. from obesity to memory deficits. Mol Neurobiol 18: 1-22. doi:10.1007/BF02741457

Ramanaviciene A, Zukiene V, Acaite J, Ramanavicius A (2002) Influence of caffeine on lysozyme activity in the blood serum of mice. Acta Med Lituanica 4: 241-244

Ramanaviciene A, Mostovojus V, Bachmatova I, Ramanavicius A (2003a) Anti-bacterial effect of caffeine on Escherichia coli and Pseudomonas fluorescens. Acta Med Lituanica 10: 185-188

Ramanaviciene A, Acaite J, Dringeliene A, Markevicius A, Ramanavicius A (2003b) Effect of caffeine on mice immunocompetent cells. Acta Med Lituanica 2: 86-89

Ribeiro JA, Sebastiao AM (2010) Caffeine and adenosine. I Alzheimers Dis 20 (Suppl 1): S3-S15. doi: 10.3233/JAD-2010-1379

Rodrigues CI, Marta L, Maia R, Miranda M, Ribeirinho M, Máguas C (2007) Application of solid-phase extraction to brewed coffee caffeine and organic acid determination by UV/HPLC. J Food Comp Anal 20: 440-448. doi: 10.1016/j.jfca.2006.08.005

Ross GW, Abbott RD, Petrovitch H, Morens DM, Grandinetti A, Tung KH, Tanner CM, Masaki KH, Blanchette PL, Curb JD, Popper JS, White LR (2000) Association of coffee and caffeine intake with the risk of Parkinson disease. JAMA 283: 2674-2679. doi: 10.1001/jama.283.20.2674

Sacks LE, Thompson PA (1977) Increased spore yields of Clostridium perfringens in the presence of methylxanthines. Appl Environ Microbiol 34: 189-93.

Sakurai H, Mitsuhashi N, Tamaki Y, Akimoto T, Murata O, Kitamoto Y, Maebayashi K, Ishikawa H, Hayakawa K, Niibe H (1999) In- teraction between low dose-rate irradiation, mild hyperthermia and low-dose caffeine in a human lung cancer cell line. Int J Radiat Biol 759: 739-745. doi: 10.1080/095530099140087

Sandlie I, Lossius I, Sjåstad K, Kleppe K (1983) Mechanism of caffeine induced inhibition of DNA synthesis in Escherichia coli. FEBS Lett 151: 237-242

Sawynok J (1995) Pharmacological rationale for the clinical use of caffeine. Drugs 49: 37-50

Selby CP, Sancar A (1990) Molecular mechanisms of DNA repair inhibition by caffeine. Proc Natl Acad Sci USA 87: 3522-3525

Suárez-Quiroz ML, González-Rios O, Barel M, Guyot B, Schorr-Galindo S, Guiraud JP (2004) Effect of chemical and environmental factors on Aspergillus ochraceus growth and toxigenesis in green coffee. Food Microbiol 21: 629-634

Tornaletti S, Russo P, Parodi S, Pedrini AM (1989) Studies on DNA binding of caffeine and derivatives: evidence of intercalation by DNA unwinding experiments. Biochim Biophys Acta 1007: 112-115

Traganos F, Kaminska-Eddy B, Darzynkiewicz Z (1991) Caffeine reverses the cytotoxic and cell kinetic effects of Novantrone (mitoxantrone). Cell Prolif 24: 305-319. doi: 10.1111/j.1365-2184.1991. tb01159.x

Veselkov DA, Kodintsev VV, Pakomor VI, Djimant LN, Davies DB, Veselkov AN (2002) 1H-NMR analysis of heteroassociation of caffeine with antibiotic actinomycin D in aqueous solutions. Biophys 45: 193-202

Vinod DR (2004) Pharmacognosy and Phytochemistry. Carrier Publication, 1 st edn, vol II, pp 311

Wilmot M (2006) In vitro inhibition of phytopathogenic fungi by caffeine, tea catechins theanine, polyphenon g, black-, green- and rooibos tea extracts. Pretoria, South Africa: Pretoria University, MSc thesis

Yen WJ, Wang BS, Chang LW, Duh PD (2005) Antioxidant properties of roasted coffee residues. I Agric Food Chem 53: 2658-2663. Doi: 10.1021/jf0402429

Youn JH, Gulve EA, Holloszy JO (1991) Calcium stimulates glucose transport in skeletal muscles by a partway independent of contraction. Am J Physiol 260: C555-C561. 